\title{
Life-form and Biological Spectrum of sub-tropical forests and agroecosystems of Manipur in North-east India
}

\author{
Sophia, M. ${ }^{1,3}$, N. Behera ${ }^{2}$ and Asha Gupta ${ }^{1}$ \\ ${ }^{1}$ School of Life Sciences, Sambalpur University, Jyoti Bihar - 768019, Sambalpur, Odisha, India \\ ${ }^{2}$ Department of Life Sciences, Manipur University, Imphal-795003, Manipur, India \\ ${ }^{3}$ Corresponding author, E-mail: sufi-amrit@yahoo.com
}

[Received 12.12.2019; Revised 27.12.2019; Accepted 28.12.2019; Published 31.12.2019]

\begin{abstract}
The present study was carried out in Community Forest (Sacred grove), Reserve Forest and Agroecosystem (Home Garden) of Imphal West district of Manipur (latitudes $23^{\circ} 50^{\prime} \mathrm{N}$ to $25^{\circ} 42^{\prime} \mathrm{N}$ and longitudes $92^{\circ} 58^{\prime} \mathrm{E}$ to $94^{\circ} 45^{\prime} \mathrm{E}$ ). A total of 159 plant species belonging to 63 families were recorded and categorized in different life forms based on the position of their perennating buds. Percentage of species belonging to each life-form category shows variation from the normal biological spectrum of Raunkiaer (1934) comprising Phanerophytes $(53.16 \%)$, Therophyte $(24.52 \%)$, Cryptophytes $(8.17 \%)$, Hemicryptophytes $(10.06 \%)$ and Chamaephytes $(5.66 \%)$. On the basis of this study the phytoclimate of the study area as per Raunkiaer's terminology, has been described as 7 phenero-therophytic phytoclimate.
\end{abstract}

Key words: Life-form, Biological spectrum, Phytoclimate, Sacred grove, Manipur

\section{INTRODUCTION}

Life-form study is an important part of vegetation description, ranking next to floristic composition (Cain 1950). Plants can be grouped in life form classes based on their similarities in structure and function (Mueller-Dombois \& Ellenberg 1974). Humboldt (1806) for the first time formulated the concept of the life forms based on the location of perennating buds or organs. According to Odland (2009) life-forms of plant are functional types that have been used to describe plant adaptation to certain ecological conditions. Plants with a similar life form are exported to exhibit similar responses on the dominant ecosystem processes (Pauses \& Austin 2001). In Raunkiaer's (1934) system, there are five major classes, arranged according to increased protection of renewing buds: Phanerophytes, Chamaephytes, Hemicryptophytes, Cryptophytes and Therophytes. According to Begon et al. (1996) it is the simplest and, in many ways, the most satisfying classification of plant life forms.

Biological spectrum is the percent representation of the number of species belonging to each life form in a given flora. Raunkiaer (1934) constructed a "normal spectrum" which could act as a null model against which different life-form spectra could be compared. Differences in the life form distribution between the normal spectrum and a biological spectrum would point out which life-form characterizes the phytoclimate or the vegetation under study.

\section{METHODOLOGY}

Three different sites were selected for the present study namely (i) Community Forest Sacred grove [Site I], located at 24046'50" N latitude and 93 ${ }^{\circ} 51^{\prime} 83^{\prime \prime}$ E longitude at Konthoujam 
village, $11 \mathrm{~km}$ west of the Imphal city, Manipur at an elevation of $781 \mathrm{~m}$ AMSL.(ii) Reserve forest [Site II], located at 24052'45" N latitude and 93 ${ }^{\circ} 54^{\prime} 51^{\prime \prime}$ E longitude at Khonghampat within the Langol Reserve Forest, $12 \mathrm{kms}$ from Imphal city at an elevation of $791 \mathrm{~m}$ AMSL and (iii) Agroecosystem, Home garden [Site III] located at 24052'31" N latitude and 93053'32" E longitude at Khamran village, $14 \mathrm{~km}$ from Imphal city at an elevation of $785 \mathrm{~m}$ AMSL. The climate of the study area experiences monsoonal climate with warm moist summer and cool dry winter. The mean maximum temperature varied from $22.5^{\circ} \mathrm{C}$ (January) to $29^{\circ} \mathrm{C}$ (September) and mean minimum temperature ranged from $4.97^{\circ} \mathrm{C}$ (January) to $22.94^{\circ} \mathrm{C}$ (July). The average annual rainfall is $1,467.5 \mathrm{~mm}$.

Intensive survey was conducted during April 2011 to March 2012 for plant collections and observation. All the species were identified and enumerated by matching at ASSAM and CAL herbaria. The voucher specimens are submitted in Life Science Department, Manipur University. The following growth forms were recognized - Trees, Shrubs and Herbs. Biological life form, habit, height and the location of perennating buds of species at three study sites were observed. The species were further placed in various life form classes as per Raunkiaer's system (Raunkiaer 1934). Biological spectrum was worked out representative of the three study sites. For categorization of species in various life forms the following formula was used:

$$
\text { Percentage of life-form }=\frac{\text { Number of species in any life-form }}{\text { Total number of species of all life-form }} \times 100
$$

\section{RESULT}

A total number of 159 species of plants belonging to 63 families were recorded from the study sites and categorized in different life forms based on the position of their perennating buds (Table 1). Families recorded with maximum species were Leguminosae, Asteraceae, Lamiaceae, Poaceae, Malvaceae, Moraceae, Myrtaceae, Lauraceae, Amaryllidaceae, Polygonaceae, Rutaceae, Solanaceae and Zingiberaceae, etc. The life form exhibited by trees and shrubs comprised of phanerophytes whereas herbs were found belonging to five major life forms viz., Phanerophytes (Ph), Chamaephytes (Ch), Hemicryptophytes (HC), Cryptophytes $(\mathrm{Cr})$ and Therophytes (Th). Biological spectrum of the three study sites is reflected in Table 2. The percentage of species belonging to each life-form category shows variation from the normal biological spectrum of Raunkiaer (1934) comprising Phanerophytes with 53.16\% as shown in Figures $1 \& 2$. Phytoclimate for the study sites as per Raunkiaer's life form classification has been described as 'phanero-therophytic' phytoclimate.

Table 1. Inventory of the floristic composition at study sites.

\begin{tabular}{|l|l|c|c|c|c|}
\hline \multicolumn{1}{|c|}{ Scientific name } & \multicolumn{1}{c|}{ Family } & $\begin{array}{c}\text { Life } \\
\text { form }\end{array}$ & $\begin{array}{c}\text { Site-I } \\
\text { (SG) }\end{array}$ & $\begin{array}{c}\text { Site-II } \\
\text { (F) }\end{array}$ & $\begin{array}{c}\text { Site-III } \\
\text { (HG) }\end{array}$ \\
\hline Abrus precatorius L. & Malvaceae & $\mathrm{Ph}$ & - & + & - \\
\hline Acacia farnesiana (L.) Willd & Leguminosae & $\mathrm{Ph}$ & - & - & + \\
\hline Achyranthes aspera L. & Amaranthaceae & $\mathrm{Cr}$ & + & - & - \\
\hline Acorus calam us L. & Acoraceae & $\mathrm{Cr}$ & - & - & + \\
\hline Adiantum aleuticum (Rupr.) C.A. Paris & Pteridaceae & $\mathrm{Hc}$ & - & + & - \\
\hline Adiantum lunulatum Burm.f. & Pteridaceae & $\mathrm{Hc}$ & + & - & - \\
\hline Aegle marmelos (L.) Correa & Rutaceae & $\mathrm{Ph}$ & + & - & - \\
\hline Ageratum conyzoides(L.) L. & Asteraceae & $\mathrm{Th}$ & + & + & - \\
\hline Albizia chinensis (Osbeck) Merr. & Leguminosae & $\mathrm{Ph}$ & + & - & - \\
\hline
\end{tabular}




\begin{tabular}{|c|c|c|c|c|c|}
\hline Scientific name & Family & $\begin{array}{l}\text { Life } \\
\text { form }\end{array}$ & $\begin{array}{l}\text { Site-I } \\
\text { (SG) }\end{array}$ & $\begin{array}{c}\text { Site-II } \\
(\mathbf{F})\end{array}$ & $\begin{array}{c}\text { Site-III } \\
\text { (HG) }\end{array}$ \\
\hline Albizia lebbeck (L.) Benth. & Leguminosae & $\mathrm{Ph}$ & - & + & - \\
\hline Allium gomphrenoides Boiss. \&Heldr. & Amaryllidaceae & $\mathrm{Cr}$ & - & - & + \\
\hline Allium hookeri Thwaites & Amaryllidaceae & $\mathrm{Cr}$ & - & - & + \\
\hline Allium ramosum $\mathrm{L}$. & Amaryllidaceae & $\mathrm{Cr}$ & - & - & + \\
\hline Alnus nepalensis D.Don & Betulaceae & $\mathrm{Ph}$ & - & + & - \\
\hline Alternanthera philoxeroides (Mart.) Griseb. & Amaranthaceae & Th & - & + & - \\
\hline Amaranthus spinosus L. & Amaranthaceae & Th & - & - & + \\
\hline Amoora rohituka (Roxb.) Wight \&Arn. & Meliaceae & $\mathrm{Ph}$ & + & - & - \\
\hline Antidesma sp. & Phyllanthaceae & $\mathrm{Ph}$ & - & + & - \\
\hline Aquilaria malaccensis Lam. & Thymelaeaceae & $\mathrm{Ph}$ & + & + & - \\
\hline Argemone maxicana $\mathrm{L}$. & Papaveraceae & Th & + & - & - \\
\hline Argyreia nervosa (Burm.f.) Bojer & Convolvulaceae & $\mathrm{Ph}$ & + & - & - \\
\hline Artabotrys hexapetalus (L.f.) Bhandari & Annonaceae & $\mathrm{Ph}$ & + & + & - \\
\hline Artocarpus integer (Thunb.) Merr. & Moraceae & $\mathrm{Ph}$ & - & - & + \\
\hline Artocarpus lakucha Buch.-Ham. & Moraceae & $\mathrm{Ph}$ & - & - & + \\
\hline Athyrium filix-femina (L.) Roth & Athyriaceae & $\mathrm{Hc}$ & - & & \\
\hline Averrhoa carambola L. & Oxalidaceae & $\mathrm{Ph}$ & - & - & + \\
\hline Bambusa nutans Wall. ex Munro & Poaceae & $\mathrm{Ph}$ & - & - & + \\
\hline Bauhinia variegata $\mathrm{L}$. & Leguminosae & $\mathrm{Ph}$ & + & - & - \\
\hline Bixa orellana L. & Bixaceae & $\mathrm{Ph}$ & - & + & - \\
\hline Blumea balsamifera (L.) DC. & Asteraceae & Th & + & - & - \\
\hline Bombax ceiba L. & Malvaceae & $\mathrm{Ph}$ & - & + & - \\
\hline Brassica oleracea L. [cultivars] & Brassicaceae & Th & - & - & + \\
\hline Brassica rapa $\mathrm{L}$. & Brassicaceae & Th & - & - & + \\
\hline Bridelia stipularis (L.) Blume & Phyllanthaceae & $\mathrm{Hc}$ & - & + & - \\
\hline Calotropis gigantean (L.)Dryand. & Apocynaceae & $\mathrm{Ph}$ & + & - & - \\
\hline Carica papaya $\mathrm{L}$. & Caricaceae & $\mathrm{Ph}$ & - & - & + \\
\hline Celtis australis L. & Cannabaceae & $\mathrm{Ph}$ & + & + & - \\
\hline Celtis timorensis Span. & Cannabaceae & $\mathrm{Ph}$ & + & - & - \\
\hline Centella asiatica (L.) Urb. & Apiaceae & $\mathrm{Hc}$ & - & - & + \\
\hline Cheilocostus speciosus (J.Koenig) C.D.Specht & Costaceae & $\mathrm{Hc}$ & + & + & - \\
\hline Chenopodium album $\mathrm{L}$. & Amaranthaceae & Th & - & - & + \\
\hline Chromolaena odorata (L.) R.M.King \& H.Rob. & Asteraceae & $\mathrm{Ph}$ & - & + & - \\
\hline Chukrasia tabularis A.Juss. & Meliaceae & $\mathrm{Ph}$ & + & - & - \\
\hline $\begin{array}{l}\text { Cinnamomum tamala (Buch.-Ham.) T.Nees \& } \\
\text { Eberm. }\end{array}$ & Lauraceae & $\mathrm{Ph}$ & - & - & + \\
\hline Cinnamomum verum J.Presl & Lauraceae & $\mathrm{Ph}$ & - & + & - \\
\hline Citrus limon (L.) Osbeck & Rutaceae & $\mathrm{Ph}$ & - & - & + \\
\hline Citrus maxima (Burm.) Merr. & Rutaceae & $\mathrm{Ph}$ & - & - & + \\
\hline Colocasia antiquorum Schott & Araceae & $\mathrm{Cr}$ & - & - & + \\
\hline Cordia grandis Roxb. & Boraginaceae & $\mathrm{Ph}$ & + & - & - \\
\hline Coriandrum sativum L. & Apiaceae & Th & - & - & + \\
\hline $\begin{array}{l}\text { Corymbia citriodora (Hook.) K.D.Hill \& } \\
\text { L.A.S.Johnson }\end{array}$ & Myrtaceae & $\mathrm{Ph}$ & - & + & - \\
\hline Crassocephalum crepidioides (Benth.) S.Moore & Asteraceae & $\mathrm{Hc}$ & - & - & + \\
\hline Crotalaria saltiana Andrews & Leguminosae & $\mathrm{Ph}$ & - & + & - \\
\hline Curcuma longa $\mathrm{L}$. & Zingiberaceae & $\mathrm{Cr}$ & - & - & + \\
\hline
\end{tabular}


Sophia, M. et al. 349

\begin{tabular}{|c|c|c|c|c|c|}
\hline Scientific name & Family & $\begin{array}{c}\text { Life } \\
\text { form }\end{array}$ & $\begin{array}{l}\text { Site-I } \\
\text { (SG) }\end{array}$ & $\begin{array}{c}\text { Site-II } \\
(\mathbf{F})\end{array}$ & $\begin{array}{c}\text { Site-III } \\
\text { (HG) }\end{array}$ \\
\hline Cyanthillium cinereum (L.) H.Rob. & Asteraceae & Th & + & - & - \\
\hline Cynodon dactylon (L.) Pers. & Poaceae & $\mathrm{Ch}$ & + & + & - \\
\hline Cynoglossum zeylanicum (Vahl) Brand & Boraginaceae & Th & + & - & - \\
\hline Cyperus rotundus $\mathrm{L}$. & Cyperaceae & $\mathrm{Cr}$ & + & + & - \\
\hline Dactyloctenium aegyptium (L.) Wild. & Poaceae & $\mathrm{Hc}$ & + & + & - \\
\hline Datura stramonium L. & Solanaceae & $\mathrm{Ph}$ & + & - & - \\
\hline Delonix regia (Boj.ex Hook.) Raf. & Leguminosae & $\mathrm{Ph}$ & + & - & - \\
\hline Derris robusta (Roxb. ex DC.) Benth. & Leguminosae & $\mathrm{Ph}$ & - & + & - \\
\hline Desmodium laxiflorum DC. & Leguminosae & $\mathrm{Th}$ & - & + & - \\
\hline Digitaria sanguinalis (L.) Scop. & Poaceae & $\mathrm{Th}$ & - & + & - \\
\hline Dipterocarpus turbinatus C.F. Gaertn & Dipterocarpaceae & $\mathrm{Ph}$ & - & + & - \\
\hline Drymaria cordata (L.) Willd.exSchult. & Caryophyllaceae & $\mathrm{Hc}$ & + & - & - \\
\hline Dryopteris filix-mas(L.) Schott & Dryopteridaceae & $\mathrm{Hc}$ & - & + & - \\
\hline Eclipta prostrata (L.) L. & Asteraceae & $\mathrm{Th}$ & + & - & - \\
\hline Elaeagnus latifolia $\mathrm{L}$. & Elaeagnaceae & $\mathrm{Ph}$ & - & - & + \\
\hline Elaeocarpus floribundus Blume & Elaeocarpaceae & $\mathrm{Ph}$ & - & - & + \\
\hline Eleusine indica (L.) Gaertn. & Poaceae & $\mathrm{Ch}$ & + & - & + \\
\hline Elsholtzia griffithii Hook.f. & Lamiaceae & Th & - & - & + \\
\hline Enydra fluctuans DC. & Asteraceae & $\mathrm{Cr}$ & - & + & + \\
\hline Eryngium foetidum $\mathrm{L}$. & Apiaceae & Th & - & - & + \\
\hline Erythrina variegata L. & Leguminosae & $\mathrm{Ph}$ & - & + & - \\
\hline Eucalyptus globules Labill & Myrtaceae & $\mathrm{Ph}$ & - & - & + \\
\hline Euphorbia hirta L. & Euphorbiaceae & $\mathrm{Th}$ & - & + & - \\
\hline Euphorbia prostrata Aiton & Euphorbiaceae & $\mathrm{Th}$ & - & + & - \\
\hline Ficus benjamina L. & Moraceae & $\mathrm{Ph}$ & + & - & - \\
\hline Ficus hispida L.f. & Moraceae & $\mathrm{Ph}$ & - & + & - \\
\hline Ficus racemosa L. & Moraceae & $\mathrm{Ph}$ & + & - & - \\
\hline Ficus religiosa L. & Moraceae & $\mathrm{Ph}$ & - & + & - \\
\hline Gmelina arborea Roxb. & Lamiaceae & $\mathrm{Ph}$ & + & + & - \\
\hline Grevillea robusta A.Cunn.ex R.Br. & Proteaceae & $\mathrm{Ph}$ & - & + & - \\
\hline Gynura cusimbua (D.Don) S. Moore & Asteraceae & $\mathrm{Th}$ & + & - & - \\
\hline Hibiscus cannabinus L. & Malvaceae & $\mathrm{Ph}$ & - & - & + \\
\hline Hibiscus rosa-sinensis L. & Malvaceae & $\mathrm{Ph}$ & - & - & + \\
\hline Houttuynia cordata Thunb. & Saururaceae & $\mathrm{Hc}$ & - & - & + \\
\hline Justicia adhatoda $\mathrm{L}$. & Acanthaceae & $\mathrm{Ph}$ & + & - & + \\
\hline Lantana camara L. & Verbenaceae & $\mathrm{Ph}$ & + & + & - \\
\hline Leucas aspera (Willd.) Link & Lamiaceae & Th & - & - & + \\
\hline Lindernia anagallis (Burm.f.) Pennell & Linderniaceae & $\mathrm{Cr}$ & - & + & - \\
\hline Lithocarpus fenestratus (Roxb.) Rehder & Fagaceae & $\mathrm{Ph}$ & - & + & - \\
\hline Litsea cubeba (Lour.) Pers. & Lauraceae & $\mathrm{Ph}$ & + & - & - \\
\hline Litsea monopetala (Roxb.) Pers. & Lauraceae & $\mathrm{Ph}$ & + & + & - \\
\hline Lolium perenne L. & Poaceae & $\mathrm{Ch}$ & - & - & + \\
\hline Luffa acutangula (L.) Roxb & Cucurbitaceae & Th & - & - & + \\
\hline Lycopersicon esculentum Mill. & Solanaceae & Th & - & - & + \\
\hline Magnolia champaca (L.) Baill. ex Pierre & Magnoliaceae & $\mathrm{Ph}$ & - & - & + \\
\hline Mangifera indica $\mathrm{L}$. & Anacardiaceae & $\mathrm{Ph}$ & + & + & - \\
\hline
\end{tabular}




\begin{tabular}{|c|c|c|c|c|c|}
\hline Scientific name & Family & $\begin{array}{l}\text { Life } \\
\text { form }\end{array}$ & $\begin{array}{l}\text { Site-I } \\
\text { (SG) }\end{array}$ & $\begin{array}{c}\text { Site-II } \\
(\mathbf{F})\end{array}$ & $\begin{array}{c}\text { Site-III } \\
\text { (HG) }\end{array}$ \\
\hline Melia azadirach $\mathrm{L}$. & Meliaceae & $\mathrm{Ph}$ & + & + & + \\
\hline Mentha piperita $\mathrm{L}$. & Lamiaceae & $\mathrm{Hc}$ & - & - & + \\
\hline $\begin{array}{l}\text { Meriandra dianthera (Roth ex Roem. \&Schult.) } \\
\text { Briq. }\end{array}$ & Lamiaceae & $\mathrm{Ph}$ & - & - & + \\
\hline Meyna laxiflora Robyns & Rubiaceae & $\mathrm{Ph}$ & - & + & - \\
\hline Mimosa pudica $\mathrm{L}$. & Leguminosae & $\mathrm{Ph}$ & + & + & - \\
\hline Musa $\times$ paradisiacal $\mathrm{L}$. & Musaceae & $\mathrm{Cr}$ & - & - & + \\
\hline Mycetia stipulate (Hook.f.) Kuntze. & Rubiaceae & $\mathrm{Ph}$ & - & + & - \\
\hline Neanotis hohenackeri P.Daniel\&Vajr. & Rubiaceae & Th & + & - & - \\
\hline Nyctanthes arbor-tristis L. & Oleaceae & $\mathrm{Ph}$ & + & - & - \\
\hline Ocimum americanum $\mathrm{L}$. & Lamiaceae & $\mathrm{Th}$ & - & - & + \\
\hline Ocimum tenuiflorum $\mathrm{L}$. & Lamiaceae & $\mathrm{Th}$ & - & - & + \\
\hline Oplismenus burmani (Retz.) P.Beauv. & Poaceae & $\mathrm{Th}$ & + & - & - \\
\hline Oroxylum indicum (L.) Kurz & Bignoniaceae & $\mathrm{Ph}$ & + & - & - \\
\hline Osbeckia stellata Buch.-Ham. ex Ker Gawl. & Melastomataceae & $\mathrm{Ph}$ & - & + & - \\
\hline Oxalis corniculata $\mathrm{L}$. & Oxalidaceae & $\mathrm{Hc}$ & - & - & + \\
\hline Parkia timoriana (DC.) Merr. & Leguminosae & $\mathrm{Ph}$ & + & + & + \\
\hline Persicaria barbata (L.) H.Hara & Polygonaceae & $\mathrm{Th}$ & - & - & + \\
\hline Persicaria chinensis (L.) H. Gross & Polygonaceae & Th & - & + & - \\
\hline Phaseolus lanatus Benth. & Leguminosae & $\mathrm{Ch}$ & - & - & + \\
\hline Phyllanthus emblica $\mathrm{L}$ & Phyllanthaceae & $\mathrm{Ph}$ & + & + & + \\
\hline Phyllanthus urinaria $\mathrm{L}$. & Euphorbiaceae & Th & - & + & - \\
\hline Pinus kesiya Royle ex Gordon. & Pinaceae & $\mathrm{Ph}$ & - & + & - \\
\hline Pisum sativum L. & Leguminosae & $\mathrm{Th}$ & - & - & + \\
\hline Plantago asiatica subsp.erosa (Wall.) Z.Yu Li & Plantaginaceae & Th & - & + & + \\
\hline Plumbago zeylanica L. & Plumbaginaceae & $\mathrm{Th}$ & - & + & - \\
\hline Plumeria rubra L. & Apocynaceae & $\mathrm{Ph}$ & - & - & + \\
\hline Polygonum posumbu Buch.-Ham. ex D.Don & Polygonaceae & Th & - & - & + \\
\hline Portulaca oleracea L. & Portulacaceae & $\mathrm{Ch}$ & + & - & - \\
\hline Psidium gujava $\mathrm{L}$. & Myrtaceae & $\mathrm{Ph}$ & - & - & + \\
\hline Punica granatum $\mathrm{L}$. & Lythraceae & $\mathrm{Ph}$ & - & - & + \\
\hline Quercus griffithii Hook.f.\& Thomson ex Miq. & Fagaceae & $\mathrm{Ph}$ & + & - & - \\
\hline Quercus serrata Murray & Fagaceae & $\mathrm{Ph}$ & - & + & - \\
\hline Ranunculus diffusus DC. & Ranunculaceae & $\mathrm{Th}$ & - & + & - \\
\hline Rhus succedanea L. & Anacardiaceae & $\mathrm{Ph}$ & - & + & - \\
\hline Ruellia prostrate Poir. & Acanthaceae & $\mathrm{Th}$ & - & + & - \\
\hline Saccharum officinarum L. & Poaceae & $\mathrm{Hc}$ & - & - & + \\
\hline Schima wallichii Choisy & Theaceae & $\mathrm{Ph}$ & - & + & - \\
\hline Scutellaria discolour Colebr. & Lamiaceae & $\mathrm{Ch}$ & - & + & - \\
\hline Sesbania sesban (L.) Merr. & Leguminosae & $\mathrm{Hc}$ & - & - & + \\
\hline Sida acuta Burm.f. & Malvaceae & $\mathrm{Ph}$ & - & + & - \\
\hline Sida cordifolia $\mathrm{L}$. & Malvaceae & $\mathrm{Ph}$ & + & - & - \\
\hline Smilax calophylla Wall. ex A.DC. & Smilacaceae & $\mathrm{Cr}$ & - & + & - \\
\hline Solanum melongena $\mathrm{L}$. & Solanaceae & $\mathrm{Ch}$ & - & - & + \\
\hline Solanum tuberosum L. & Solanaceae & $\mathrm{Th}$ & - & - & + \\
\hline Spilanthes acmella (L.) L. & Asteraceae & Th & + & - & - \\
\hline Spondias macrophylla Klotzsch ex Engl. & Anacardiaceae & $\mathrm{Ph}$ & - & + & - \\
\hline
\end{tabular}




\begin{tabular}{|l|l|c|c|c|c|}
\hline \multicolumn{1}{|c|}{ Scientific name } & \multicolumn{1}{c|}{ Family } & $\begin{array}{c}\text { Life } \\
\text { form }\end{array}$ & $\begin{array}{c}\text { Site-I } \\
\text { (SG) }\end{array}$ & $\begin{array}{c}\text { Site-II } \\
\text { (F) }\end{array}$ & $\begin{array}{c}\text { Site-III } \\
\text { (HG) }\end{array}$ \\
\hline Stachytarpheta indica (L) Vahl & Verbenaceae & $\mathrm{Ch}$ & + & - & - \\
\hline Stellaria media (L.) Vill. & Caryophyllaceae & $\mathrm{Ch}$ & - & - & + \\
\hline Syzygium cymosum (Lam.) DC. & Myrtaceae & $\mathrm{Ph}$ & + & - & - \\
\hline Syzygium jambos (L.) Alston & Myrtaceae & $\mathrm{Ph}$ & + & - & - \\
\hline Taraxacum campylodes G.E.Haglund & Asteraceae & $\mathrm{Ph}$ & - & + & - \\
\hline Tectona grandis L.f. & Lamiaceae & $\mathrm{Ph}$ & + & + & - \\
\hline Terminalia chebula Retz. & Combretaceae & $\mathrm{Ph}$ & + & - & - \\
\hline Toona ciliata M. Roem. & Meliaceae & $\mathrm{Ph}$ & + & + & + \\
\hline Tridax procumbens (L.) L. & Asteraceae & $\mathrm{Th}$ & + & - & - \\
\hline Urena lobata L. & Malvaceae & $\mathrm{Ph}$ & - & + & - \\
\hline Vitex negundo L. & Lamiaceae & $\mathrm{Ph}$ & + & + & - \\
\hline Xylosma longifolia Clos & Salicaceae & $\mathrm{Ph}$ & - & + & - \\
\hline Zingiber officinale Roscoe & Zingiberaceae & $\mathrm{Cr}$ & - & - & + \\
\hline
\end{tabular}

Table 2.Comparison of biological spectrum of study sites with Raunkiaer's (1934) Normal Biological Spectrum.

\begin{tabular}{|l|c|c|}
\hline \multirow{2}{*}{$\begin{array}{c}\text { Life-form } \\
\text { class }\end{array}$} & \multicolumn{2}{|c|}{ Life form Percentage (\%) } \\
\cline { 2 - 3 } & Normal Spectrum & Present study \\
\hline PH & 46.0 & 52.83 \\
\hline CH & 9.0 & 5.66 \\
\hline H & 26.0 & 9.43 \\
\hline CR & 6.0 & 8.18 \\
\hline TH & 13.0 & 23.90 \\
\hline
\end{tabular}

Table 3. Comparison of Biological Spectrum of the present study sites with other subtropical climatic types in Manipur.

\begin{tabular}{|c|c|c|c|c|c|c|}
\hline Location & PH & $\mathbf{C H}$ & $\mathbf{H}$ & $\mathbf{C R}$ & TH & Reference \\
\hline $\begin{array}{l}\text { Normal Biological } \\
\text { Spectrum of Raunkiaer }\end{array}$ & 46.0 & 9.0 & 26.0 & 6.0 & 13.0 & $\begin{array}{c}\text { Raunkiaer } \\
(1934)\end{array}$ \\
\hline $\begin{array}{l}\text { Sub-tropical forest } \\
\text { Manipur }\end{array}$ & 66.9 & 8.06 & 4.03 & 4.83 & 16.1 & $\begin{array}{l}\text { Usharani } \\
(2004)\end{array}$ \\
\hline $\begin{array}{l}\text { Teak Dipterocarpus } \\
\text { forest, Indo-Myanmar } \\
\text { border Site I }\end{array}$ & 63.2 & 8.1 & 2.04 & 6.12 & 20.4 & $\begin{array}{l}\text { Ranita et al. } \\
\text { (2010) }\end{array}$ \\
\hline Site II & 65.9 & 8.5 & 2.12 & 4.25 & 19.1 & \\
\hline Hill forest of Manipur & 62.76 & 10.64 & 1.06 & 7.45 & 18.08 & $\begin{array}{l}\text { Devi et al. } \\
\text { (2014) }\end{array}$ \\
\hline $\begin{array}{l}\text { Amambilok Sacred } \\
\text { Grove, Andro, Manipur }\end{array}$ & 55.41 & 6.36 & 10.19 & 8.28 & 19.74 & $\begin{array}{c}\text { Imosana \& } \\
\text { Gupta (2015) }\end{array}$ \\
\hline $\begin{array}{l}\text { Subtropical Montane } \\
\text { forest Site I }\end{array}$ & 53.49 & 10.46 & 11.62 & 8.14 & 16.28 & $\begin{array}{c}\text { Easter \&Gupta } \\
(2016)\end{array}$ \\
\hline $\begin{array}{l}\text { Temperate Montane } \\
\text { forest, Site II }\end{array}$ & 63.64 & 9.09 & 6.81 & 18.19 & 2.27 & \\
\hline $\begin{array}{l}\text { Subtropical } \\
\text { Phytoclimate }\end{array}$ & 52.83 & 5.66 & 9.43 & 8.18 & 23.90 & Present study \\
\hline
\end{tabular}

\section{DISCUSSION}

Biological spectrum not only represents the climatic conditions but the most potent environmental factor representing the ecosystem (Pandeya \& Sharma 1950). Similar phyto- 


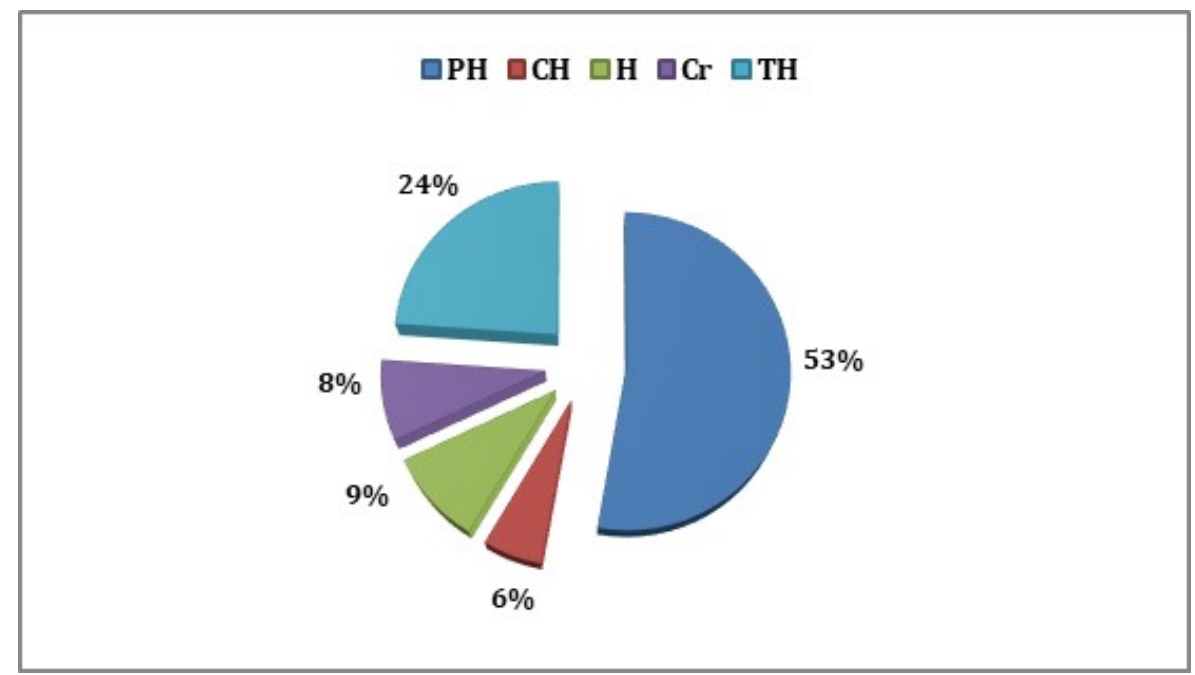

Figure 1.Graphical representation of different Life forms in study sites.

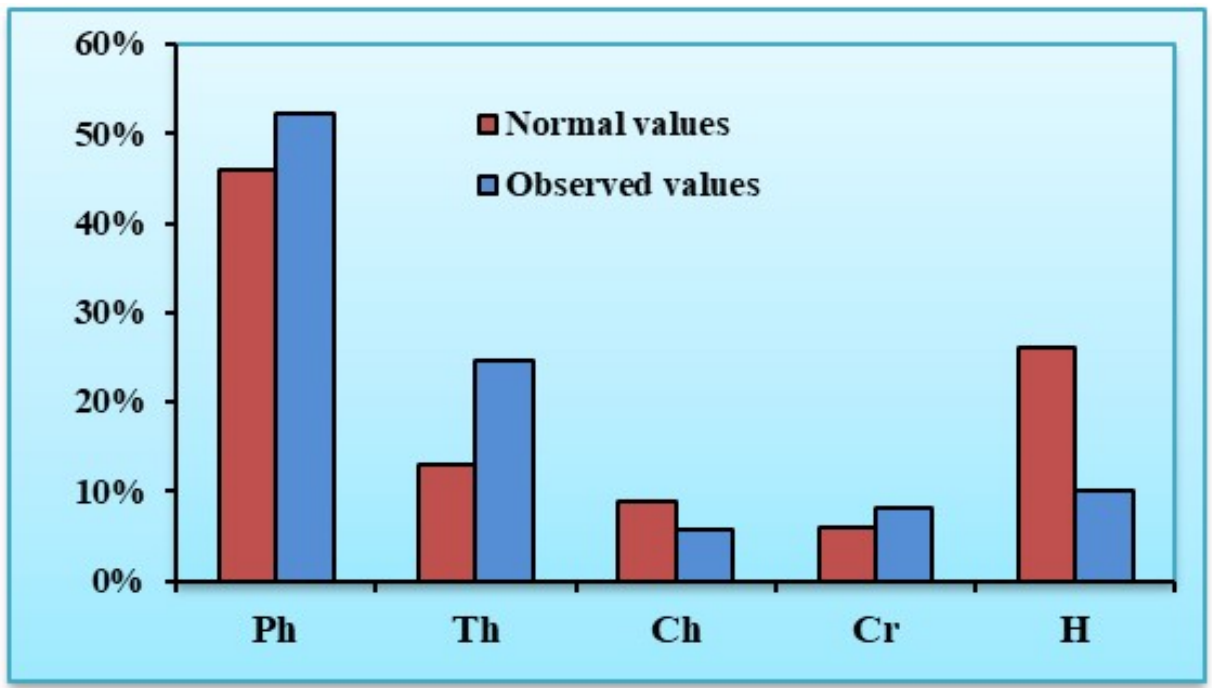

Figure 2.Comparison of biological spectrum of study sites (Average value) with Raunkiaer's Normal Spectrum.

climatic conditions have also been reported by some workers (Lakshmanan 1962; Misra et al. 1979; Saxena \& Singh 1980; Khatri 2000; Devi et al. 2014; Imosana \& Devi 2015; Easter \& Devi 2016). Biological life forms are reflected by bioclimate of the area (MeherHomji 1964). Thus, in humid regions, the bioclimate should be phanerophytic, in arid regions and intensively cultivated areas as therophytic and in temperate high altitudinal zones, arctic regions as chamaephytic. According to Subramani et al. (2007) higher percentage of Therophytes occurring in the area is the characteristic of subtropics and often related to soil and climatic conditions. It has also been reported that Therophytes and next to Phanerophytes (Thakur 2015). Prevalence of Therophytes is also an indicator of biotic pressure (Bharucha $\&$ Dave 1944). The occurrence of Therophytes was much favoured in disturbed areas (Keeley \& Albert 1977; Vora \& George 1987). Dominance of Phanerophytes reveal the 
significance of trees in ameliorating the micro-climate, controlling the regeneration, establishment of herbaceous plants, maintenance of biodiversity and functioning of the ecosystem.

\section{CONCLUSION}

The phytoclimate of the present study revealed that the vegetation is predominantly subtropical type having a higher percentage of Phanerophytes and Therophytes indicates influence of anthropogenic activities which favors the growth of short lived annuals.

\section{Acknowledgements}

The authors are thankful to the Manipur Forest Department for granting site permissions during the research period and also thanks to the local people residing near the study area for their kind co-operation during the study.

\section{LITERATURE CITED}

Begon, M.; Harper, J.L. \& Townsend, C.R. 1996. Ecology: Individuals, Populations and Communities. Blackwell, Oxford.

Bharucha, F.R. \& Dave, R.N. 1944. The biological spectrum of Grassland association.J. Univ. Bombay. 13: $15-25$.

Cain, S.A. 1950. Life-forms and Phytoclimate. Bot. Rev. 16: 1 - 32.

Devi, K.B.; Singh, H.B. \& Gupta, A. 2014. Life form in Hill Forest of Manipur, North-East, India. J. Agroeco. Nat. Res. Manag. 1(1): 7 - 13.

Easter, K.A. \& Gupta, A. 2016. Life-forms and biological spectrum along the altitudinal gradient in Montane Forests of Senapati district of Manipur in North-East india. Pleione 10(1): $80-89$.

Humboldt, A. von 1806. Ideen zu einer Physiognomik der Gewächse. Tübingen: Cotta. [Ideas for a physiognomy of plants, pp. 210-352]

Imosana, R.K. \& Gupta,A. 2015. Life-form classification and biological spectrum of Amambiliok Sacred Grove, Andro, Manipur in Northeast India. Pleione 9(2): 356 - 364.

Keeley, S.C. \& Albert, W.J. 1977. A comparision of pattern of herb, shrub growth in comparable sites of chili and California. Am. Midl. Natur. 97: 120 - 132.

Khatri, P.K. 2000. Study on biodiversity in tropical forest ecosystem of Satpura National Park Madhya Pradesh. Ph.D. Thesis. Forest Research institute, Dehra Dun.

Lakshmanan, N.K. 1962. The application of Raunkiaer's life-forms. J. Indian Bot. Soc. 41: $585-589$.

Meher-Homji, V.M. 1964. Life forms and biological spectra as epharmonic criteria of aridity and humidity in tropics.J. Indian Bot. Soc. 43(3): $424-430$.

Misra, M.P.; Mishra, P.N. \& Pandey, B.N. 1979. An ecological account of the vegetation of Brahmyoni Hills (Gaya). Proc. Indian Sci. Cong. 3: 108 - 109.

Mueller-Dombois, D. \& Ellenberg, H. 1974. Aims and methods of vegetation ecology. John Wiley and Sons, New York.

Odland, A. 2009. Interpretation of altitudinal gradient in South Central Norway based on vascular plants as environmental indicators. Ecol. Indicators. 9: 409 - 421. 
Pandey, S.C. \& Sharma, I.K.V. 1950. The biological spectrum of the flora of Makronia Plateau. Bull. Bot. Soc., Univ. Saugor: 22.

Pausas, J.G. \& Austin, M.P. 2001. Pattern of plant species richness in relation to different environments.An appraisal. J. Veg. Sci. 12: $153-166$.

Ranita, N.; Kandya, A.K. \& Gupta, A. 2010. Composition, Diversity and Distribution Trends in Human Impacted Indo-Myanmar Border Forest. Proceedings on National Conference on Current Scenario on Global Challenges in Germplasm Conservation and Biodiversity, Nagpur University. 17 - 18.

Raunkiaer, C. 1934. The life forms of plants and statistical plant geography. Claredon Press, Oxford.

Saxena, A.K. \& Singh, J.S. 1980. "Analysis of forest grazing land and vegetation in parts of Kumuan Himalaya land" J. Range Mange, 1: 13 - 32.

Subramani, S.P.; Jishtu, V.; Verma, R.K. \& Kapoor, K.S. 2007. Floristic composition, life forms and biological spectrum of Renuka Wild life Sanctuary, Himachal Pradesh.Indian For. 133: $79-92$.

Thakur, A.S. 2015. Floristic composition, life-forms and biological spectrum of tropical dry deciduous forest in Sagar District, M.P. India. Trop. Pl. Res. 2(2): $112-119$.

Usharani, L. 2004. Ecological studies of structure and functioning of certain sacred groves of Manipur, North-East India. Ph.D. Thesis, Manipur University.

Vora, A.B. \& George, V.C. 1987. The distribution of various life forms in the ground flora under different canopies of Panchamahal forests, Gujarat, Indian J. For. 10: $223-225$. 